\title{
RenderGAN: Generating Realistic Labeled Data
}

\author{
Leon Sixt*, Benjamin Wild and Tim Landgraf \\ Fachbereich Mathematik und Informatik, Freie Universität Berlin, Berlin, Germany
}

Deep Convolutional Neuronal Networks (DCNNs) are showing remarkable performance on many computer vision tasks. Due to their large parameter space, they require many labeled samples when trained in a supervised setting. The costs of annotating data manually can render the use of DCNNs infeasible. We present a novel framework called RenderGAN that can generate large amounts of realistic, labeled images by combining a 3D model and the Generative Adversarial Network framework. In our approach, image augmentations (e.g., lighting, background, and detail) are learned from unlabeled data such that the generated images are strikingly realistic while preserving the labels known from the 3D model. We apply the RenderGAN framework to generate images of barcode-like markers that are attached to honeybees. Training a DCNN on data generated by the RenderGAN yields considerably better performance than training it on

OPEN ACCESS

Edited by:

Guanghui Wang,

University of Kansas, United States

Reviewed by:

Zhichao Lian,

Nanjing University of Science and

Technology, China

Xinlei Chen

Facebook, United States

*Correspondence:

Leon Sixt

leon.sixt@fu-berlin.de

Specialty section:

This article was submitted to Vision Systems Theory, Tools and

Applications,

a section of the journal Frontiers in Robotics and Al

Received: 01 February 2018 Accepted: 17 May 2018 Published: 08 June 2018

Citation:

Sixt L, Wild B and Landgraf T (2018) RenderGAN: Generating Realistic Labeled Data. Front. Robot. Al 5:66. doi: 10.3389/frobt.2018.00066 various baselines.

Keywords: generative adversarial networks, unsupervised learning, social insects, markers, deep learning

\section{INTRODUCTION}

When an image is taken from a real-world scene, many factors determine the final appearance: background, lighting, object shape, position, and orientation of the object, the noise of the camera sensor, and more. In computer vision, high-level information such as class, shape, or pose is reconstructed from raw image data. Most real-world applications require the reconstruction to be invariant to noise, background, and lighting changes.

In recent years, deep convolutional neural networks (DCNNs) advanced to the state of the art in many computer vision tasks (Krizhevsky et al., 2012; Razavian et al., 2014; He et al., 2015). More training data usually increases the performance of DCNNs. While image data is mostly abundant, labels for supervised training must often be created manually-a time-consuming and tedious activity. For complex annotations such as human joint angles, camera viewpoint, or image segmentation, the costs of labeling can be prohibitive.

In our project BeesBook (Wario et al., 2015), we extract and analyze the social network of a honeybee colony. The bees are monitored with multiple cameras and can be identified by a unique binary marker attached on their back. To track the bees, it is required to decode the binary marker reliably. The recent successes of DCNNs (Russakovsky et al., 2015) implied that a DCNN could decode bees markers if enough training samples are available. However, annotating an image of a single marker takes about a minute. If we labeled only 10 images for each of the 4,096 different IDs, we would have to work more than $680 \mathrm{~h}$, i.e., several months of tedious labor. It would still be questionable if 10 samples could reliably represent the image variance due to different lighting conditions and object rotations in space. Thus, the main question is how can we acquire more labeled data? 
A simple solution could have been to annotate more data by crowdsourcing the task. This would likely have created several months of work for distributing the data, and collecting and processing the labels. Another intuitive approach would be to train a DCNN on images generated by a $3 \mathrm{D}$ renderer. We used a simple 3D model (no lighting, shading) but could not train a DCNN which performed well on real image data.

Here we propose a solution based on a modification to the recently proposed GAN framework (Goodfellow et al., 2014). We use a simple 3D model of the binary marker to generate an idealistic image of the marker. The generator network then learns to add the missing image detail such as lighting, background, and image noise so that the resulting images look strikingly real. By ensuring that the generator cannot change the ID label, we can collect realistic images from the generator with their corresponding labels we fed to the $3 \mathrm{D}$ model. This dataset can then be used to train a DCNN which performs well on real data.

In the next section, we shortly review the state-of-the-art in generating realistic images. Subsequently, we describe our RenderGAN method and compare the results of a DCNN trained to decode the binary marker with different baselines. Finally, we discuss our results in the BeesBook project, and in a wider context of generating realistic data with preserved labels.

\section{RELATED WORK}

A common approach to deal with limited amount of labels is data augmentation (Goodfellow et al., 2016, Chapter 7.4). Translation, noise, and other deformations can often be applied without changing the labels, thereby effectively increasing the number of training samples and reducing overfitting. Ratner et al. (2017) propose to automatically learn augmentations with GANs.

DCNNs learn a hierarchy of features-many of which are applicable to related domains (Yosinski et al., 2014). Therefore, a common technique is to pre-train a model on a larger dataset such as ImageNet (Deng et al., 2009) and then fine-tune its parameters to the task at hand (Girshick et al., 2014; Razavian et al., 2014; Long et al., 2015). This technique only works in cases where a large enough related dataset exists. Furthermore, labeling enough data for fine-tuning might still be costly.

If a basic model of the data exists (for example, a 3D model of the human body), it can be used to generate labeled data. Peng et al. (2015) generated training data for a DCNN with 3DCAD models. Su et al. (2015) used 3D-CAD models from large online repositories to generate large amounts of training images for the viewpoint estimation task on the PASCAL 3D+ dataset (Xiang et al., 2014). Zhou et al. (2016) also use the PASCAL 3D+ dataset to learn the dense flow prediction between images. $\mathrm{Wu}$ et al. (2016) construct a network to learn a 3D skeleton of objects such as chairs. Massa et al. (2015) are matching natural images to $3 \mathrm{D}-\mathrm{CAD}$ models with features extracted from a DCNN. Richter et al. (2016) and Ros et al. (2016) used 3D game engines to collect labeled data for image segmentation. However, the explicit modeling of the image acquisition physics (scene lighting, reflections, lense distortions, sensor noise, etc.) is cumbersome and might still not be able to fully reproduce the particularities of the imaging process such as unstructured background or object specific noise. Training a DCNN on generated data that misses certain features will result in overfitting and poor performance on the real data.

Generative Adversarial Networks (GAN) (see Figure 1) can learn to generate high-quality samples (Goodfellow et al., 2014), i.e., sample from the data distribution $p(x)$. Denton et al. (2015) synthesized images with a GAN on the CIFAR dataset (Krizhevsky, 2009), which were hard for humans to distinguish from real images. While a GAN implicitly learns a meaningful latent embedding of the data (Radford et al., 2015), there is no simple relationship between the latent dimensions and the labels of interest. Therefore, high-level information can't be inferred from generated samples. cGANs are an extension of GANs to sample from a conditional distribution given some labels, i.e., $p(x \mid l)$. However, training cGANs requires a labeled dataset. Springenberg (2015) showed that GANs can be used in a semi-supervised setting but restricted their analysis to categorical labels. Wang and Gupta (2016) trained two separate GANs, one to model the object normals and another one for the texture conditioned on the normals. As they rely on conditional GANs, they need large amounts of labeled data. Chen et al. (2016) used an information theoretic to disentangle the representation. They decomposed the representation into a structured and unstructured part. And successfully related on a qualitative level the structured part to high-level concepts such as camera viewpoint or hair style. However, explicitly controlling the relationship between the latent space and generated samples without using labeled data is an open problem, i.e., sampling from $p(x, l)$ without requiring labels for training.

Independent work by Shrivastava et al. (2016) proposes to postprocess images of a $3 \mathrm{D}$ model of eyes and hand poses with a GAN. In contrast to our work, they propose a L1-loss to ensure that the labels remain valid.

Planar markers with binary codes have been shown to be feasible for tracking large groups of insects. A system, previously developed for ants (Mersch et al., 2013) was shown to successfully track 100 bees for 2 days Blut et al. (2017). The markers in used were originally described as fiducial markers in augmented reality systems (Fiala, 2005) and rely on spatial derivatives to detect the rectangular outline of a tag. A similar system using flat and rectangular markers for tracking larger insects was also proposed and might be adapted to honeybees (Crall et al., 2015). This

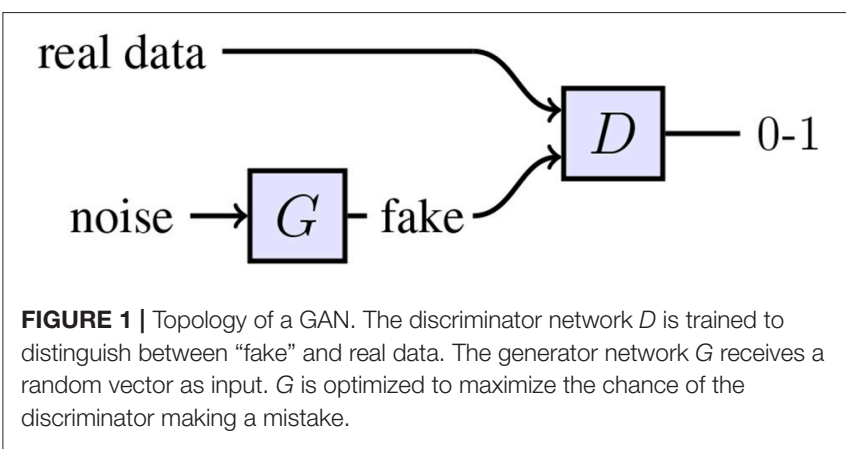


system binarizes the image globally and searches for rectangular regions representing the corners of the marker. The previous BeesBook vision system (Wario et al., 2015) was tailored to specifically track all animals of small honeybee colonies over their entire lifetime. It uses a round and curved marker and searches for ellipse-shaped edge formations.

\section{RENDERGAN}

In the BeesBook project (Wario et al., 2015), we need to decode the position, orientation and binary id of markers attached to honeybees in a hive. Due to the large amount of honeybees this task can't be done manually. Although we invested a substantial amount of time on labeling, a DCNN trained on the limited labeled dataset did not perform well. We therefore wanted to synthesize labeled images which are realistic enough to train an improved decoder network.

\subsection{D Model}

We use a 3D Model to generate an idealistic image of the marker. The $3 \mathrm{D}$ model comprises a mesh which represents the structure of the marker and a simple camera model to project the mesh to the image plane (see Figure 2). The model is parameterized by its position, pitch, yaw and roll, and bit assignment. Given a parameter set, the $3 \mathrm{D}$ model is rendered to an image of the marker, a background segmentation mask and a depth map. The generated images is an idealistic representation of the marker

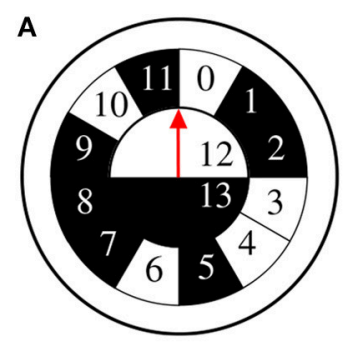

Marker structure

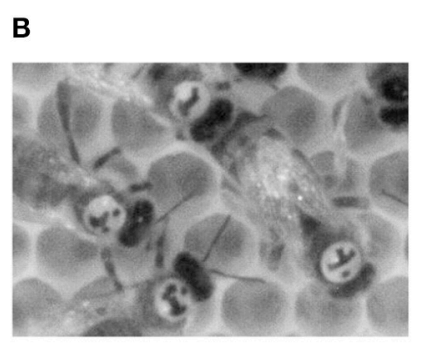

Tagged bees in the hive
FIGURE 2 | (A) The marker represents a unique binary code (cell 0-11) and encodes the orientation with the semicircles 12 and 13. The red arrow points toward the head of the bee. This marker encodes the id 100110100010 . (B) Cutout from a high-resolution image. and lacks many important factors: blur, lighting, background, and image detail (see Figure 3). A DCNN trained on data from this idealistic model did not generalize to real images.

The discriminator gradients cannot be backpropagated through the 3D model. Still, we want to learn the distributions of the inputs of the $3 \mathrm{D}$ model such as the orientation and position. While there exists differentiable renders (Loper and Black, 2014), we found it the easiest to train a neural network to emulate the $3 \mathrm{D}$ model. Its outputs are indistinguishable from the images of the $3 \mathrm{D}$ model. Yet, the neural network allows backpropagation of the gradients. The weights of the $3 \mathrm{D}$ model network are fixed during the GAN training. The bit assignments are sampled uniformly.

\subsection{Augmentations}

Normally augmentations are used to enlarge and diversify the training data. Typical augmentations are translation, rotation, adding noise, and scaling of pixel intensities. The parameters of the augmentations are normally sampled from a random distribution. Here, one has to ensure that the augmentations don't change the content of the image. For example, adding too much noise can occlude the object.

In the RenderGAN, we would like to learn the parameters to a set of augmentation such that a simple $3 \mathrm{D}$ model is morphed to a realistic image. We have to ensure that all augmentations preserve the high-level information.

We apply different augmentations that account for blur, lighting, background, and image detail. The output of the $3 \mathrm{D}$ model and of each augmentation is of the same shape as the image. In Figure 4, the structure of the generator is shown.

\subsubsection{Blurriness}

The 3D model produces hard edges, but the images of the real tags show a broad range of blur. The generator produces a scalar $\alpha \in[0,1]$ per image that controls the blur.

$$
\phi_{b l u r}(x, \alpha)=(1-\alpha)\left(x-b_{\sigma}(x)\right)+b_{\sigma}(x)
$$

where $b_{\sigma}(x)=x * k_{\sigma}$ denotes convolving the image $x$ with a Gaussian kernel $k_{\sigma}$ of scale $\sigma$. The implementation of the blur function is inspired by Laplacian pyramids (Burt and Adelson, 1983). As required for augmentations, the labels are preserved, because we limit the maximum amount of blur by picking $\sigma=2$. $\phi_{\text {blur }}$ is also differentiable w.r.t the inputs $\alpha$ and $x$.

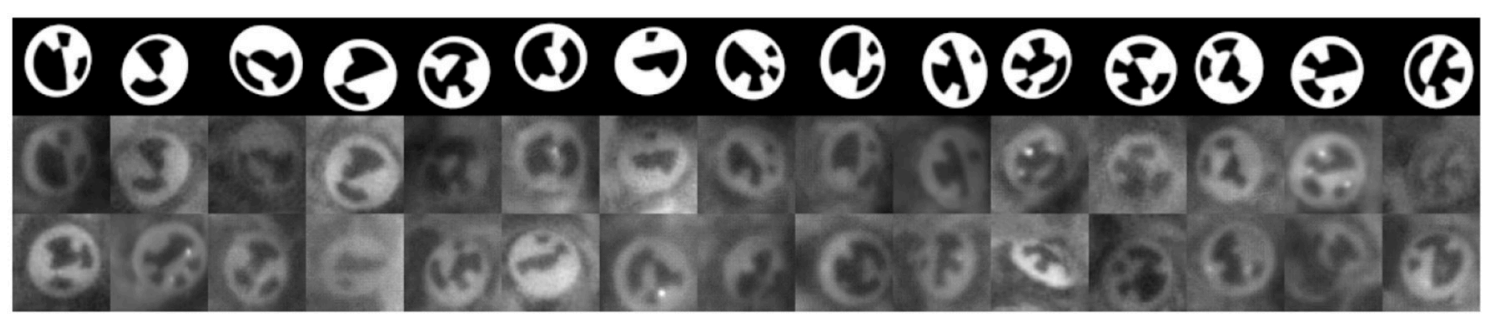

FIGURE 3 | First row: Images from the 3D model without augmentation. Below: Corresponding images from the RenderGAN. Last row: Real images of bee's markers. 


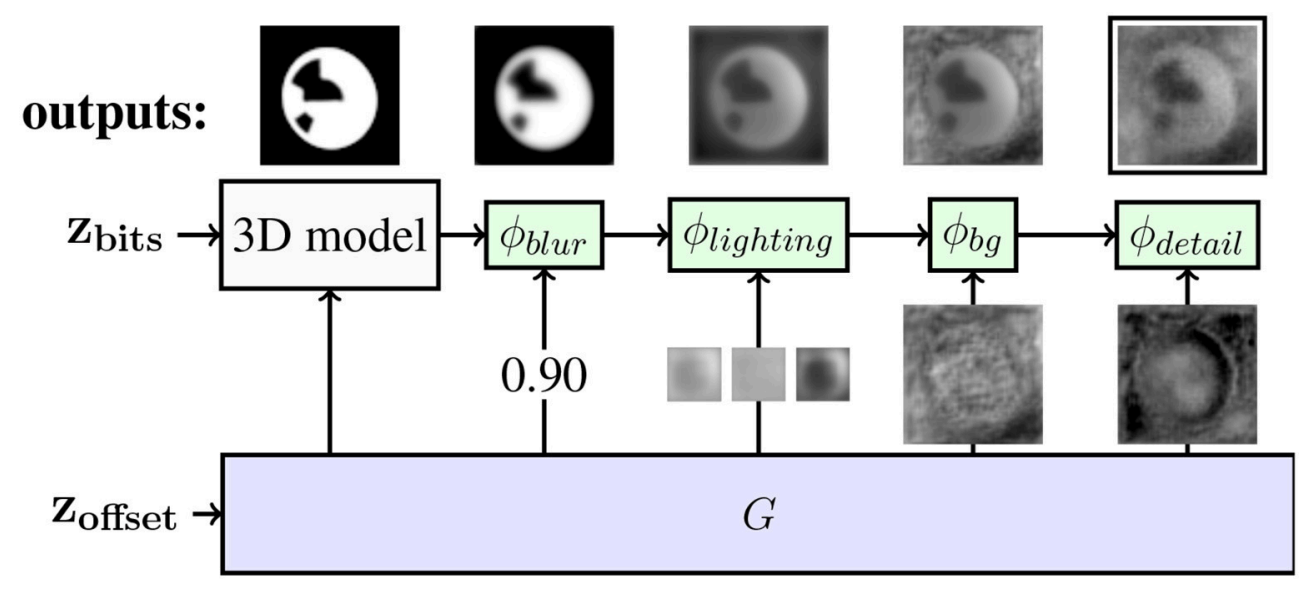

FIGURE 4 | Augmentations of the RenderGAN applied to the BeesBook project. The arrows from $G$ to the augmentations $\phi$ depict the inputs to the augmentations. The generator provides the position and orientations to the 3D model, whereas the bits are sampled uniformly. On top, the output of each stage is shown. The output of $\phi_{\text {detail }}$ is forwarded to the discriminator.

\subsubsection{Lighting of the Tag}

The images from the $3 \mathrm{D}$ model are binary. In real images, tags exhibit different shades of gray. We model the lighting by a smooth scaling and shifting of the pixel intensities. The generator provides three outputs for the lighting: scaling of black parts $s_{b}$, scaling of white parts $s_{w}$ and a shift $t$. All outputs have the same dimensions as the image $x$. An important invariant is that the black bits of the tag must stay darker than the white bits. Otherwise, a bit could flip, and the label would change. By restricting the scaling $s_{w}$ and $s_{b}$ to be between 0.10 and 1 , we ensure that this invariant holds. The lighting is locally corrolated and should cause smooth changes in the image. Hence, Gaussian blur $b(x)$ is applied to $s_{b}, s_{w}$, and $t$.

$\phi_{\text {lighting }}\left(x, s_{w}, s_{b}, t\right)=x \cdot b\left(s_{w}\right) \cdot W(x)+x \cdot b\left(s_{b}\right) \cdot(1-W(x))+b(t)$

The segmentation mask $W(x)$ is one for white parts and zero for the black part of the image. As the intensity of the input is distributed around -1 and 1 , we can use thresholding to differentiate between black and white parts.

\subsubsection{Background}

The background augmentation can change the background pixels arbitrarily. A segmentation mask $B_{x}$ marks the background pixels of the image $x$ which are replaced by the pixels from the generated image $d$.

$$
\phi_{b g}(x, d)=x \cdot\left(1-B_{x}\right)+d \cdot B_{x}
$$

The 3D model provides the segmentation mask. As $\phi_{b g}$ can only change background pixels, the labels remain unchanged.

\subsubsection{Details}

In this stage, the generator can add small details to the whole image including the tag. The output of the generator $d$ is passed through a high-pass filter to ensure that the added details are small enough not to flip a bit. Furthermore, $d$ is restricted to be in $[-2,2]$ to make sure the generator cannot avoid the highpass filter by producing huge values. With the range $[-2,2]$, the generator has the possibility to change black pixels to white, which is needed to model spotlights.

$$
\phi_{\text {detail }}(x, d)=x+\operatorname{highpass}(d)
$$

The high-pass is implemented by taking the difference between the image and a blurred version of the image $(\sigma=3.5)$. As the spotlights on the tags are only a little smaller than the bits, we increase its slope after the cutoff frequency by repeating the high-pass filter three times.

The image augmentations are applied in the order as listed above: $\phi_{\text {detail }} \circ \phi_{\text {background }} \circ \phi_{\text {lighting }} \circ \phi_{\text {blur }}$. Please note that there exist parameters to the augmentations that could change the labels. As long as it is guaranteed that such augmentations will result in unrealistic looking images, the generator network will learn to avoid them. For example, even though the detail augmentation could be used to add high-frequency noise to obscure the tag, this artifact would be detected by the discriminator.

\subsection{Technical Details}

\subsubsection{Architecture of the Generator}

The generator network has to produce outputs for each augmentation. Here, we outline the most important parts. In Appendix E (Supplementary Material), we show how the network modules are connected and list all layers. The generator starts with a small network consisting of dense layers, which predicts the parameters for the $3 \mathrm{D}$ model (position, orientations). The output of another dense layer is reshaped and used as starting block for a chain of convolution and upsampling layers. We found it advantageous to merge a depth map of the 3D model into the generator as especially the lighting depends on the orientation of the tag in space. The input to the blur augmentation is predicted by reducing an intermediate convolutional feature map 
to a single scalar. An additional network is branched off to predict the input to the lighting augmentation. For the background generation, the output of the lighting network is merged back into the main generator network together with the actual image from the 3D model.

For the discriminator architecture, we mostly rely on the architecture given by Radford et al. (2015), but doubled the number of convolutional layers and added a final dense layer. This change improved the quality of the generated images.

\subsubsection{Clip Layer}

Some of the augmentation parameters have to be restricted to a range of values to ensure that the labels remain valid. The training did not converge when using functions like tanh or sigmoid due to vanishing gradients. We are using a combination of clipping and activity regularization to keep the output in a given interval $[a, b]$. If the input $x$ is out of bounds, it is clipped and a regularization loss $r$ depending on the distance between $x$ and the appropriate bound is added.

$$
\begin{aligned}
& r(x)= \begin{cases}\gamma\|x-a\|_{1} & \text { if } x<a \\
0 & \text { if } a \leq x \leq b \\
\gamma\|x-b\|_{1} & \text { if } x>b\end{cases} \\
& f(x)=\min (\max (a, x), b)
\end{aligned}
$$

With the scalar $\gamma$, the weight of the loss can be adapted. For us $\gamma=15$ worked well. If $\gamma$ is chosen too small, the regularization loss might not be big enough to move the output of the previous layer toward the interval $[a, b]$. During training, we observe that the loss decreases to a small value but never vanishes.

\subsubsection{Training}

We train generator and discriminator as in the normal GAN setting. We use 2.4 M unlabeled images of tags to train the RenderGAN. We use Adam (Kingma and Ba, 2014) as an optimizer with a starting learning rate of 0.0002 , which we reduce in epoch 200, 250, and 300 by a factor of 0.25 . In Figure $5 \mathbf{B}$ the training loss of the GAN is shown. The GAN does not converge to the point where the discriminator can no longer separate generated from real samples. There is also a difference between how real and fake tags are scored by the discriminator after training (see Figure 5A). The augmentation might restrict the generator too much such that it cannot model certain properties.
Nevertheless, it is hard for a human to distinguish the generated from real images. In some cases, the generator creates unrealistic high-frequencies artifacts. The discriminator unfailingly assigns a low score to theses images. We can therefore discard them for the training of the supervised algorithm. More generated images are shown in Appendix A (Supplementary Material). In Figure 6, we show random points in the latent space, while fixing the tag parameters. The generator indeed learned to model the various lighting conditions, noise intensities, and backgrounds.

\section{RESULTS}

We constructed the RenderGAN to generate labeled data. But does a DCNN trained with the RenderGAN data perform better than one trained on the limited amounts of real data? And are learned augmentations indeed needed or do simple handdesigned augmentation achieve the same result? The following paragraphs describe the different datasets used in the evaluation. We focus on the performance of a DCNN on the generated data. Thus, we do not compare our method to conventional GANs as those do not provide labels and are generally hard to evaluate.

\subsection{Data From the RenderGAN}

We generate 5 million tags with the RenderGAN framework. Due to the abundance, one training sample is only used twice during training. It is not further augmented.

\subsection{Real Data}

The labels of the real data are extracted from ground truth data that was originally collected to evaluate bee trajectories. This ground truth data contains the path and id of each bee over multiple consecutive frames. Data from five different time spans was annotated-in total $66 \mathrm{~K}$ tags. As the data is correlated in time (same ids, similar lighting conditions), we assign the data from one time span completely to either the train or test set. The data from three time spans forms the train set $(40 \mathrm{~K})$. The test set $(26 \mathrm{~K})$ contains data from the remaining two time spans. The ground truth data lacks the orientation of the tags, which is therefore omitted for this evaluation. Due to the smaller size of the real training set, we augment it with random translation, rotation, shear transformation, histogram scaling, and noise (see Appendix C in Supplementary Material for exact parameters).
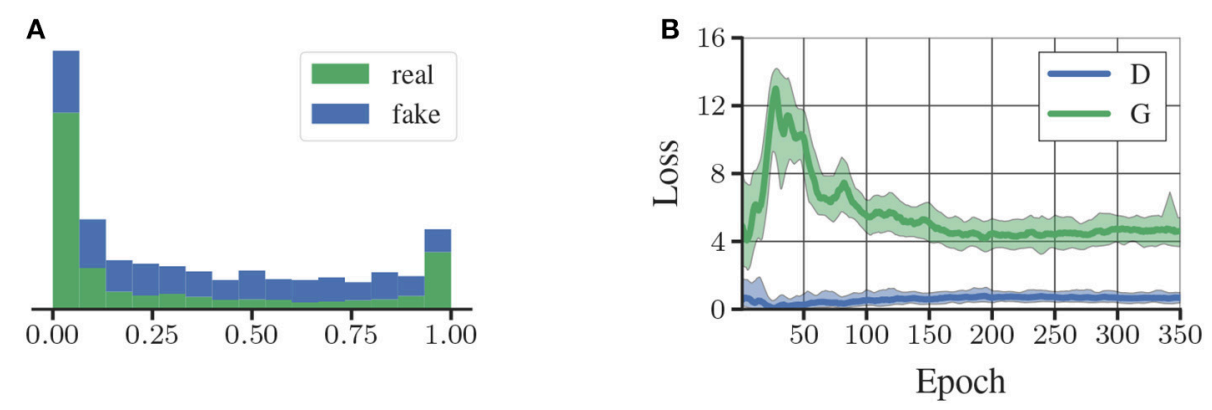

FIGURE 5 | (A) Histogram of the discriminator scores on fake and real samples. (B) Losses of the generator (G) and discriminator (D). 


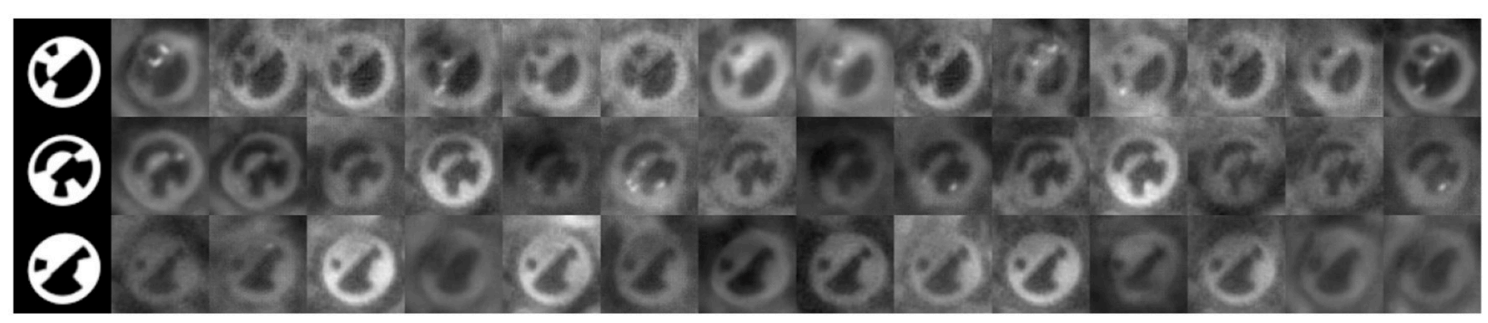

FIGURE 6 | Random points in the z-space given the tag parameters.

TABLE 1 | Datasets created with learned representations and hand-designed augmentations.

\begin{tabular}{lll}
\hline Name & Learned & Hand-Designed \\
\hline HM 3D & 3D model & $\begin{array}{l}\text { Blur, lighting, background, } \\
\text { noise, spotlights } \\
\text { HM LI }\end{array}$ \\
HM BG & 3D model, blur, lighting & $\begin{array}{l}\text { Background, noise, } \\
\text { spotlights }\end{array}$ \\
& background & Noise, spotlights \\
\end{tabular}

\subsection{RenderGAN + Real}

We also train a DCNN on generated and real data which is mixed at a 50:50 ratio.

\subsection{Handmade Augmentations}

We tried to emulate the augmentations learned by the RenderGAN by hand. For example, we generate the background by an image pyramid where the pixel intensities are drawn randomly. We model all effects, i.e., blur, lighting, background, noise, and spotlights (see Appendix B in Supplementary Material for details on their implementation). When sampling the 5 million images from the RenderGAN, we also save the simple tags and all between steps which are then the input to the augmentations. The augmentations are applied on the fly during training. Therefore, we can rule out that the amount of training samples is responsible for the performance differences. We apply the handmade augmentation to different learned representations of the RenderGAN, e.g., we use the learned lighting representation and add the remaining effects such as background and noise with handmade augmentations ( $H M$ $L I)$. See Table 1 for the different combinations of learned representations and hand designed augmentations.

\subsection{Computer Vision Pipeline CV}

The previously used computer vision pipeline (Wario et al., 2015) is based on manual feature extraction. For example, a modified Hough transformation to find ellipses. The MHD obtained by this model is only a rough estimate given that the computer vision pipeline had to be evaluated and fine-tuned on the same data set due to label scarcity.

\subsection{Training Setup}

An epoch consists of 1,000 batches á 128 samples. We use early stopping to select the best parameters of the networks. For the training with generated data, we use the real training set as the validation set. When training on real data, the test set is also used for validation. We could alternatively reduce the real training set further and form an extra validation set, but this would harm the performance of the DCNN trained on the real data. We use the 34-layer ResNet architecture (He et al., 2015) but start with 16 feature maps instead of 64 . The DCNNs are evaluated on the mean Hamming distance (MHD) i.e., the expected value of bits decoded wrong. Human experts can decode tags with a MHD of around 0.23 .

\subsection{Results}

In Table 2, we present the results of the evaluation. The training losses of the networks are plotted in Figure 7. The model trained with the data generated by the RenderGAN has an MHD of 0.424 . The performance can furthermore be slightly improved by combining the generated with real data. The small gap in performance when adding real data is a further indicator of the quality of the generated samples.

If we use predictions from this DCNN instead of the computer vision pipeline, the accuracy of the tracking improves from 55\% of the ids assigned correctly to $96 \%$. At this quality, it is possible to analyze the social behavior of the honeybees reliably.

Compared to the handmade augmentations (HM 3D), data from the RenderGAN leads to considerably better performance. The large gap in performance between the HM 3D and HM LI data highlights the importance of the learned lighting augmentation.

\section{DISCUSSION}

We proposed a novel extension to the GAN framework for adding realism to a simplistic $3 \mathrm{D}$ object model. Compared to computer graphics pipelines, the RenderGAN can learn complex effects from unlabeled data that would be otherwise hard to model with explicit rules. Contrary to the many variants of GANs, the generator provides explicit information about the synthesized images, which can be used as labels for supervised learning. The training of the RenderGAN requires no labels.

In our project BeesBook, RenderGAN was able to generate very realistic images of bee markers. A decoder DCNN trained 
TABLE 2 | Comparison of the mean Hamming distance (MHD) on the different data sets. More samples of the training data can be found in Appendix D (Supplementary Material).

\begin{tabular}{|c|c|c|}
\hline Data & MHD & Training data \\
\hline Real & 0.956 & \\
\hline HM 3D & 0.820 & \\
\hline HM LI & 0.491 & \\
\hline HM BG & 0.505 & \\
\hline RenderGAN & 0.424 & \\
\hline RenderGAN + Real & 0.416 & 3 \\
\hline
\end{tabular}

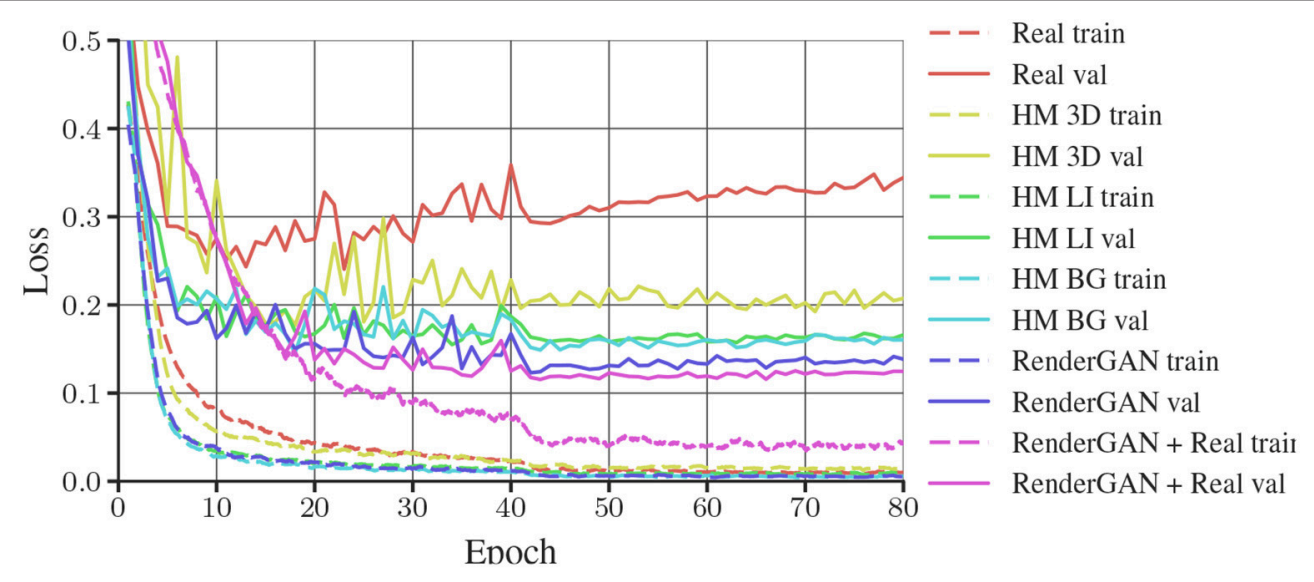

FIGURE 7 | Training and validation losses of DCNNs trained on different data sets. As some data sets are missing the orientation of the tags, only the loss of the bits are plotted. Binary crossentropy is used as loss for the bits. The train and validation loss of each dataset have the same color.

on this data outperforms decoders trained on various baselines. Compared to the previously used non-neural algorithm (Wario et al., 2015) RenderGAN improved the decoding accuracy significantly. Consequently the downstream tracking process that links detections through time improved in several respects. For the BeesBook project, RenderGAN was a key enabler. Without accurate ID decodings, a much larger portion of trajectories (and behaviors that we predict from them) would be assigned incorrect IDs. We have now created the largest database of honeybee trajectories consisting of approximately 4,000 animals, followed over a nine weeks recording period.

We believe that RenderGAN might be applicable to similar problems in other domains. The specific requirements of the BeesBook project can be generalized to many popular settings. Many applications could benefit from a decoder network that extracts high level properties that are costly to label, such as the pose of cars, facial features in portrait photos, or the body posture of pedestrians. Most computer vision algorithms in these domains rely already on an object model. Applying RenderGAN would then only require an additional definition of appropriate augmentations which can model the domain specific image artifacts. Some augmentations described here, such as background and the highpass filter, are very general and could be useful in other areas. Once RenderGAN is set up, arbitrary amounts of labeled data can be acquired with no further effort.

Furthermore, the RenderGAN approach can save substantial amounts of time if the data distribution changes. For example, if we would alter the marker design in the BeesBook project to include more bits, only small adaptations to the $3 \mathrm{D}$ model's source code and potentially some hyperparameters of the augmentations would be sufficient. In contrast, every change in object design would require new sessions of manual labeling. 


\section{FUTURE WORK}

For future work, it would be interesting to see the RenderGAN framework used on other tasks, e.g., human faces, pose estimation, or viewpoint prediction. In different contexts, different augmentations may be required, for example, colorization, affine transformations, or diffeomorphism. The RenderGAN could be especially valuable to domains where pre-trained models are not available or when the annotations are complex. Another direction of future work might be to extend the RenderGAN framework to other fields. For example, in speech synthesis, one could use an existing software synthesizer as a basic model and improve the realism of the output with a similar approach as in the RenderGAN framework.

\section{ETHICS STATEMENT}

German law does not require approval of an ethics committee for studies involving insects.

\section{REFERENCES}

Blut, C., Crespi, A., Mersch, D., Keller, L., Zhao, L., Kollmann, M., et al. (2017). Automated computer-based detection of encounter behaviours in groups of honeybees. Sci. Rep. 7:17663. doi: 10.1038/s41598-017-17863-4

Burt, P., and Adelson, E. (1983). The laplacian pyramid as a compact image code. IEEE Trans. Commun. 31, 532-540.

Chen, X., Duan, Y., Houthooft, R., Schulman, J., Sutskever, I., and Abbeel, P. (2016). Infogan: interpretable representation learning by information maximizing generative adversarial nets. arXiv preprint arXiv:1606. 03657.

Crall, J. D., Gravish, N., Mountcastle, A. M., and Combes, S. A. (2015). BEEtag: a low-cost, image-based tracking system for the study of animal behavior and locomotion. PLOS ONE 10:e0136487. doi: 10.1371/journal.pone.01 36487

Deng, J., Dong, W., Socher, R., Li, L.-J., Li, K., and Fei-Fei, L. (2009). "Imagenet: a large-scale hierarchical image database," in IEEE Conference on Computer Vision and Pattern Recognition, 2009. CVPR 2009 (Miami Beach, FL), 248-255.

Denton, E., Chintala, S., Szlam, A., and Fergus, R. (2015). "Deep generative image models using a laplacian pyramid of adversarial networks," in Advances in Neural Information Processing Systems (Montréal, QC), 1486-1494.

Fiala, M. (2005). "ARTag, a fiducial marker system using digital techniques," in 2005 IEEE Computer Society Conference on Computer Vision and Pattern Recognition (CVPR'05), Vol. 2 (San Diego, CA), 590-596.

Girshick, R., Donahue, J., Darrell, T., and Malik, J. (2014). "Rich feature hierarchies for accurate object detection and semantic segmentation," in Proceedings of the IEEE Conference on Computer Vision and Pattern Recognition (Columbus, $\mathrm{OH}$ ), 580-587.

Goodfellow, I., Bengio, Y., and Courville, A. (2016). Deep Learning. Book in preparation for MIT Press.

Goodfellow, I., Pouget-Abadie, J., Mirza, M., Xu, B., Warde-Farley, D., Ozair, S., et al. (2014). "Generative adversarial nets," in Advances in Neural Information Processing Systems (Montréal, QC), 2672-2680.

He, K., Zhang, X., Ren, S., and Sun, J. (2015). Deep residual learning for image recognition. arXiv preprint arXiv:1512.03385.

Kingma, D., and Ba, J. (2014). Adam: a method for stochastic optimization. arXiv preprint arXiv:1412.6980.

Krizhevsky, A. (2009). Learning Multiple Layers of Features from Tiny Images.

Krizhevsky, A., Sutskever, I., and Hinton, G. E. (2012). "Imagenet classification with deep convolutional neural networks," in Advances in Neural Information Processing Systems (Lake Tahoe), 1097-1105.
AUTHOR CONTRIBUTIONS

LS, BW, and TL: conceptualization, data curation, writingoriginal draft, writing-review and editing; LS: methodology, software, visualization; TL: resources, supervision, project administration.

\section{ACKNOWLEDGMENTS}

We acknowledge support by the Open Access Publication Fund of the Freie Universität Berlin. The BeesBook project was supported by the Northgerman Supercomputing Alliance (HLRN), project reference beb00002.

\section{SUPPLEMENTARY MATERIAL}

The Supplementary Material for this article can be found online at: https://www.frontiersin.org/articles/10.3389/frobt. 2018.00066/full\#supplementary-material

Long, J., Shelhamer, E., and Darrell, T. (2015). "Fully convolutional networks for semantic segmentation," in Proceedings of the IEEE Conference on Computer Vision and Pattern Recognition (Boston, MA), 3431-3440.

Loper, M. M., and Black, M. J. (2014). "Opendr: an approximate differentiable renderer," in European Conference on Computer Vision (Zürich: Springer), $154-169$.

Massa, F., Russell, B., and Aubry, M. (2015). Deep exemplar 2d-3d detection by adapting from real to rendered views. arXiv preprint arXiv:1512.02497.

Mersch, D. P., Crespi, A., and Keller, L. (2013). Tracking individuals shows spatial fidelity is a key regulator of ant social organization. Science 340, 1090-1093. doi: $10.1126 /$ science. 1234316

Peng, X., Sun, B., Ali, K., and Saenko, K. (2015). Learning deep object detectors from 3D models. ICCV (Santiago).

Radford, A., Metz, L., and Chintala, S. (2015). Unsupervised representation learning with deep convolutional generative adversarial networks. arXiv:1511.06434.

Ratner, A. J., Ehrenberg, H. R., Hussain, Z., Dunnmon, J., and Ré, C. (2017). Learning to compose domain-specific transformations for data augmentation. arXiv preprint arXiv:1709.01643.

Razavian, A. S., Azizpour, H., Sullivan, J., and Carlsson, S. (2014). "Cnn features off-the-shelf: an astounding baseline for recognition," in Proceedings of the IEEE Conference on Computer Vision and Pattern Recognition Workshops (Columbus, $\mathrm{OH}), 806-813$.

Richter, S. R., Vineet, V., Roth, S., and Koltun, V. (2016). "Playing for data: ground truth from computer games," in European Conference on Computer Vision (Amsterdam: Springer), 102-118.

Ros, G., Sellart, L., Materzynska, J., Vazquez, D., and Lopez, A. M. (2016). "The synthia dataset: a large collection of synthetic images for semantic segmentation of urban scenes," in Proceedings of the IEEE Conference on Computer Vision and Pattern Recognition (Las Vegas, NV), 3234-3243.

Russakovsky, O., Deng, J., Su, H., Krause, J., Satheesh, S., Ma, S., et al. (2015). ImageNet large scale visual recognition challenge. Int. J. Comput. Vis. 115, 211-252. doi: 10.1007/s11263-015-0816-y

Shrivastava, A., Pfister, T., Tuzel, O., Susskind, J., Wang, W., and Webb, R (2016). Learning from simulated and unsupervised images through adversarial training. arXiv preprint arXiv:1612.07828.

Springenberg, J. T. (2015). Unsupervised and semi-supervised learning with categorical generative adversarial networks. arXiv preprint arXiv:1511.06390.

Su, H., Qi, C. R., Li, Y., and Guibas, L. J. (2015). "Render for CNN: viewpoint estimation in images using cnns trained with rendered $3 \mathrm{~d}$ model views," in Proceedings of the IEEE International Conference on Computer Vision (Santiago), 2686-2694. 
Wang, X., and Gupta, A. (2016). Generative image modeling using style and structure adversarial networks. arXiv preprint arXiv:1603.05631.

Wario, F., Wild, B., Couvillon, M. J., Rojas, R., and Landgraf, T. (2015). Automatic methods for long-term tracking and the detection and decoding of communication dances in honeybees. Front. Ecol. Evol. 3:103. doi: $10.3389 /$ fevo.2015.00103

Wu, J., Xue, T., Lim, J. J., Tian, Y., Tenenbaum, J. B., Torralba, A., et al. (2016). "Single image 3d interpreter network," in European Conference on Computer Vision (Springer), 365-382.

Xiang, Y., Mottaghi, R., and Savarese, S. (2014). "Beyond pascal: a benchmark for $3 \mathrm{~d}$ object detection in the wild," in IEEE Winter Conference on Applications of Computer Vision, 75-82.

Yosinski, J., Clune, J., Bengio, Y., and Lipson, H. (2014). "How transferable are features in deep neural networks?" in Advances in Neural Information Processing Systems (Montréal, QC), 3320-3328.
Zhou, T., Krahenbuhl, P., Aubry, M., Huang, Q., and Efros, A. A. (2016). “Learning dense correspondence via 3d-guided cycle consistency," in Proceedings of the IEEE Conference on Computer Vision and Pattern Recognition (Las Vegas, NV), $117-126$.

Conflict of Interest Statement: The authors declare that the research was conducted in the absence of any commercial or financial relationships that could be construed as a potential conflict of interest.

Copyright (c) 2018 Sixt, Wild and Landgraf. This is an open-access article distributed under the terms of the Creative Commons Attribution License (CC BY). The use, distribution or reproduction in other forums is permitted, provided the original author(s) and the copyright owner are credited and that the original publication in this journal is cited, in accordance with accepted academic practice. No use, distribution or reproduction is permitted which does not comply with these terms. 